\title{
Infected hepatic cyst complicating urinary sepsis
}

\author{
A. Coulon*, I. Ould-Nana*, J-Y. Wilputte \\ Department of Gastro-enterology, Clinique Saint-Joseph, Arlon, Belgium.
}

\begin{abstract}
Congenital hepatic cysts are a common disorder. Usually they are asymptomatic and do not have to be treated. However, some serious complications can occur. We report here the case of an 86year old patient who has been treated by a percutaneous drainage for an infected solitary hepatic cyst due to urinary sepsis. She was admitted to the Department of Internal Medicine for epigastric pain with fever and chills. The patient was treated for a urinary tract infection 3 weeks ago by her General Practitioner. On admission, blood tests showed 21620 neutrophils per microliter with a C-reactive protein level at $443.7 \mathrm{mg} / \mathrm{L}$, procalcitonin $>200 \mathrm{ng} / \mathrm{mL}$, total bilirubin at $1.43 \mathrm{mg} / \mathrm{dL}$, lactate dehydrogenase at $666 \mathrm{U} / \mathrm{L}$ and alanine aminotransferase at $227 \mathrm{U} / \mathrm{L}$. Urinalysis and hemocultures highlighted the presence of Escherichia coli and Streptococcus constellatus. The abdominal tomodensitometry indicated the presence of a left hepatic biliary cyst with banal appearance. She was first treated with intravenous amoxicillin clavulanic acid. After a few days, another abdominal tomodensitometry with contrast pinpointed a large abscess of 11 centimeters in diameter extending to liver segments II and IV with a similar small lesion in segments IV and V. clindamycin per os was added to the treatment because of its good diffusion in tissues. Percutaneous drain was inserted under tomodensitometric control and stayed in place until the follow-up at three weeks. Bacteriologic culture on the fluid sample demonstrated the presence of Escherichia coli and Streptococcus constellatus. The abscess completely regressed after 6 weeks of treatment and the biologic abnormalities resolved at the same time. (Acta gastroenterol. belg., 2021, 84, 131-134).
\end{abstract}

Keywords : infected hepatic cyst, urinary sepsis, hepatic abscess, percutaneous drainage

\section{Introduction}

Solitary hepatic cyst is a benign finding but can be complicated occasionally by rupture, intracystic haemorrhage, obstructive jaundice or infection (1).

We report here the case of an 86-year old Caucasian woman who has been treated for an infected solitary hepatic cyst secondary to urinary sepsis with systemic antibiotherapy and abscess drainage. The antibiotics (amoxicillin clavulanic acid and clindamycin) used in this case are unusual but were able to treat efficiently this patient with a complete regression of the abscesses and a full recovery of the patient.

\section{Case report}

An 86-year-old retired Caucasian woman was admitted to the Department of Internal Medicine for an 8-days history of epigastric pain with fever up to $39.1^{\circ} \mathrm{C}$ and chills. Pain increased with meal and the patient complained about appetite because of this.

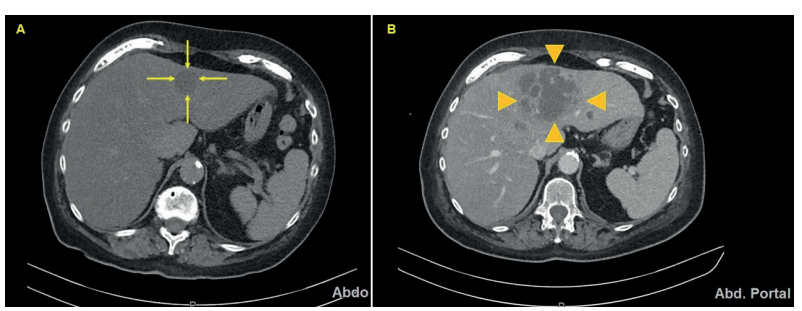

Figure 1. - A : showing abdominal tomodensitometry without contrast (Emergency Room) - left hepatic biliary cyst of banal appearance (arrow). B : showing abdominal tomodensitometry repeated 4 days later with contrast because of an uncontrolled sepsis - large abscess of $11 \mathrm{~cm}$ in diameter extending to liver segments II and IV (arrow's head).

The patient was treated with Nitrofurantoin $100 \mathrm{mg}$ three times per day for an infection of the urinary tract (Escherichia coli in the urinary sample) three weeks earlier by her General Practitioner. The treatment lasted for five days.

On physical examination, only a right hypochondral sensibility was observed. Peristaltism was perceived as normal and the cardiologic and pulmonary examination was without any particularity.

Blood tests on admission showed 21620 neutrophils

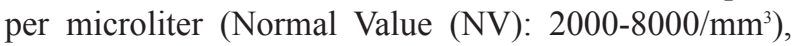
C-reactive protein level at $443.7 \mathrm{mg} / \mathrm{L}(\mathrm{NV}<5 \mathrm{mg} / \mathrm{L})$, urea at $109 \mathrm{mg} / \mathrm{dL}$ (NV: 24-60), creatinine at $1.91 \mathrm{mg} / \mathrm{dL}$ (NV: $0.5-1.1 \mathrm{mg} / \mathrm{dL})$, total bilirubin at $1.43 \mathrm{mg} / \mathrm{dL}(\mathrm{NV}$ : $0.2-1$ ), conjugated bilirubin at $0.64 \mathrm{mg} / \mathrm{dL}$ (NV : $0.1-$ $0.4)$, lactate dehydrogenase at $666 \mathrm{U} / \mathrm{L}(\mathrm{NV}: 135-215)$, alanine aminotransferase at $227 \mathrm{U} / \mathrm{L}(\mathrm{NV}<40)$, aspartate aminotransferase at $182 \mathrm{U} / \mathrm{L}(\mathrm{NV}<37)$, gamma glutamyl transferase at $128 \mathrm{U} / \mathrm{L}(\mathrm{NV}: 5-36)$, troponine $\mathrm{T}$ at 0.263 $\mathrm{ng} / \mathrm{mL}(\mathrm{NV}<0.014)$ and procalcitonin $>200 \mathrm{ng} / \mathrm{mL}(\mathrm{NV}$ $<0.5$ ). The rest of the blood tests, e.g. haemoglobin and ionogram, were without particularity. Both urinalysis and hemocultures highlighted the presence of Escherichia coli and Streptococcus constellatus.

The abdominal tomodensitometry without contrast performed in the Emergency Department indicated only the presence of a left hepatic biliary cyst of banal

\footnotetext{
Correspondence to : Ismaïl Ould-Nânâ, Department of Gastro-enterology, Cliniques Universitaires Saint-Luc, Brussels, Belgium.

E-mail : ismail.ouldnana@gmail.com

* These authors contributed equally to this work.

Submission date : 02/02/2020

Acceptance date : 15/04/2020
} 
appearance (Figure 1A. Arrow). Other finding was a simple diverticulosis without any signs of diverticulitis. There was no sign of pyelonephritis.

A close monitoring was implemented because of the severe sepsis highlighted by the blood tests. An empirical intravenous antibiotic treatment with amoxicillin clavulanic acid, $1 \mathrm{~g}$ three times a day due to the renal insufficiency, was initiated. It was based on the antibiogram of Escherichia coli in the urinary culture made by the general practitioner

Since hemocultures stayed positive, CT scan was repeated with contrast four days later to search for a potential origin of the ongoing sepsis. This second CT scan showed a large abscess of 11 centimeters in diameter extending to liver segments II and IV (Figure 1B. arro's head).

A CT-guided drainage was performed with a drain left in place. Bacteriologic culture on the fluid sample highlighted again the presence of Escherichia coli and Streptococcus constellatus.

Gastroscopy and colonoscopy during hospitalisation were unremarkable. They were performed after the second to be sure that there is not another origin for the sepsis like a digestive neoplasia.

Clindamycin per os, at the dosis of $600 \mathrm{mg}$ three times per day, was empirically added to the treatment four days after her admission. This supplementary antibiotic was choosen based on the second CT-scanner performed four days after her admission and because of its good diffusion in abscess. Furthermore, with a bitherapy we had fewer probabilities to develop bacterial resistance against our therapeutic arsenal.

Abdominal tomodensitometry with contrast was then performed once a week to follow the abscess's diameter. The abscess drain was removed after three weeks. The abscess completely regressed after 6 weeks of treatment and the biologic abnormalities resolved at the same time.

The diagnosis of an infected hepatic cyst caused by bacteraemia consecutive to a urinary tract infection was retained based on the clinical history and the results of urinary cultures and hemocultures.

\section{Discussion}

Congenital hepatic cysts are common in the population (prevalence ranges from $2 \%$ to $4 \%$ ), are usually asymptomatic and do not have to be treated. These cysts are mostly described by patients between 40 and 60 years old but can be found at any age (1). There is a femaleto-male ratio of $1,5: 1$ (2). The localization is more common in the right lobe $(2 \mathrm{x})$ than the left and rarely in the falciform ligament. If there is no communication with the biliary tree, it is considered as a simple hepatic cyst.

Some serious complications can occur such as infection $(3 \%$ when there is an autosomal dominant polycystic kidney disease (ADPKD) with end-stage renal failure and $<1 \%$ when it is milder renal failure (3)), rupture in the pericardium of an infected hepatic cyst (once described by a patient with polycystic liver and renal transplantation), fistulisation, compression of nearby structures and intracystic haemorrhage. However, in another study, they found that there was a lack of epidemiological information about infection of hepatic cysts in patients with ADPKD (4). Another study in ADKPD patients reports that $91 \%$ of them had at least one liver cyst and 12\% complex hepatic cysts (defined as haemorrhagic and/or infected cysts) but there was no precise prevalence about infected hepatic cysts (5).

In this case, our patient did not have any history or familial history of autosomal dominant polycystic kidney disease. In the literature, there are some case reports but we could not find any specific information about the exact prevalence for patients who do not suffer from ADPKD. Thus, it seems that this complication is underestimated (6).

The typical symptoms in this disease are febrile or subfebrile condition with right upper quadrant pain. Other symptoms include also nausea and vomiting, weight loss, anorexia, weakness and, less frequently, diaphragmatic irritation causing cough and/or pain in the right shoulder $(1,8)$. Leucocytosis, erythrocyte sedimentation with high rate and mild liver-function impairment can be seen in the blood tests.

Infected hepatic cysts can be caused by intraabdominal infections because of the portal spread of the germ such as appendicitis, colitis, diverticulitis (which are common etiology). Haematogenous dissemination is a cause of infected hepatic cysts such as pyelonephritis or endocarditis. Other causes of dissemination is through the biliary tree (and biliary obstruction), traumatism or infected liver tumors $(8,9)$. Iatrogenic infections, for example after an aspiration sclerotherapy of a hepatic cyst, appear as well (10).

The predominant type of infection is monomicrobial in patients with ADPKD (Escherichia coli) (7) but polymicrobial when the patients have noncystic hepatic abscesses. In our patient, it was actually a polymicrobial infection and the abdominal CT-scan did not highlight any ADKPD. There was also no familial history. Causal germs are anaerobes, Staphylococci, Streptococcus milleri, Escherichia coli, Neisseria and Yersinia enterocolitica (9). In this case, our patient had a urinary sepsis (Escherichia coli and Streptococcus constellatus) and the abdominal tomodensitometry excluded any complicated diverticulitis.

Based on the history of recent urinary tract infection, fever, urinary culture and hemoculture positive with the same germs in the fluid sample of the surinfected hepatic cyst, the cause of this infected hepatic cyst was probably a pyelonephritis without radiologic signs. There were no radiologic signs of pyelonephritis because of the tomodensitometry was performed 8 days after the first episode of fever and the patient was already treated with furadantin. Even if an image of multiple diverticulosis was seen on the computed tomography, the same exam has not highlighted any sign of diverticulitis. Therefore, 
the urinary infection (Escherichia coli and Streptococcus constellatus) as the origin of the infected congenital cyst was retained, which is an uncommon cause.

The modern imaging techniques allow us to diagnose rapidly these lesions in the liver (ultrasonography, abdominal CT-scanner) (8). In this case, CT-scanner showed us first a left hepatic biliary cyst of banal appearance and then a large abscess attending the liver segments II and IV with small lesion in the IVth and Vth segments.

The typical image of hepatic cysts on CT is a hypoattenuated and homogeneous lesion without any enhancement after intravenous injection of contrast material (11). The typical image on ultrasonography for simple hepatic cysts is an anechoic cyst. If there is a wall thickening, it suggests complications such as infection or haemorrhage (12). On the MRI, in T1-weighted sequence, the simple hepatic cyst will be a rounded, homogeneous and hypointense lesion. In T2-weighted sequence, it will be hyperintense (13).

There are two phases that can be seen in an infected hepatic cyst (9). The first one is the pre-suppurative one where the cyst appears to be heterogeneous, hypodense and irregular contours on the CT-scanner. The second phase, the suppurative phase, is characterized by an anechogenic or hypogenic cyst and a wall thickening on ultrasonography. On a contrast tomodensitometry, we can visualize the "ring sign" which is a peripheral enhancement of the infected cyst without any central enhancement. The presence of internal gas bubbles is quasi-pathognomic. However, this sign can also be seen after certain procedures (e.g. hepatic artery embolization, surgery) or when there is a communication with the digestive tract.

The ${ }^{18} \mathrm{~F}$-FDG PET/CT is a useful imaging technique to differentiate renal cyst infection from hepatic cyst infection (7). However, our patient did not have any history or familial history of autosomal dominant polycystic kidney disease and in our case, this imaging technique was unnecessary.

Because of the possible fatal complications (peritonitis due to rupture, sepsis, ...), the primary treatment is through systemic antibiotics (8). In hepatic abscesses, the empirical antibiotic treatment targets Gram-positive cocci and aerobic Gram-negative bacilli (e.g. amoxicillinclavulanic acid, piperacillin-tazobactam, third generation of cephalosporin) but also should cover anaerobes as well (e.g. metronidazole) $(9,14)$. The treatment should be adapted to the sensitivity of causative organisms. In our case, the initial diagnosis was a urinary sepsis due to the history of the previous urinary infection treated by her general practitioner. When the final diagnosis had been made, information on its causative germs and their sensitivity was available. Based on this antibiogram and the favorable evolution of the patient, antibiotic treatment based on amoxicillin and clavulanic acid associated with clindamycin was pursued.

When this patient was admitted, there were no signs of an infected hepatic cyst. Therefore there was a delay in diagnosis and unusual antibiotic therapy. In this case, the recommended antibiotic therapy would not have changed the prognosis due to germs in the hemoculture and the urinary culture. In our case, we proposed a different antibiotic therapy, based first on the urinary culture (Escherichia coli) then on the antibiogram of the responsible germs (amoxicillin clavulanic acid and clindamycin). However, after 6 weeks, the abscesses completely regressed with full recovery of the patient.

The other recommended treatment for infected cyst is a percutaneous drainage in addition to these systemic antibiotics. The size criteria for drainage are under debate. However, it seems that an abscess larger than $3 \mathrm{~cm}$ should be drained $(14,15)$. The drainage has to be realized within 24 hours of presentation although some case reports suggest other criteria such as continuous pyrexia after 48$72 \mathrm{~h}$ of efficiency antibiotherapy, suspicion of perforation (clinically and ultrasonographically) (16). We conclude to three criteria for drainage : large abscess, presence of gas, severe sepsis (17). In this case, there were two of the three-cited criteria (severe sepsis and large lesion).

The drain removal is suggested when there is a decrease in size or diminution of the drainage and with a clinical improvement.

Surgical drainage was the standard before the percutaneous drainage techniques raised (minimally invasive approach) even though there are still few indications for surgical intervention (8).

The uniqueness of this case we have reported here is the origin of the infected hepatic cysts. In the literature, there are very few reports of such cases. One was described as an infected hepatic cyst. The only reported case demonstrating the possibility of a renal infective pathology spreading to the liver is that of a pyelo-hepatic fistula following xanthogranulomatous pyelonephritis by Chung et al. (18).

\section{Conclusion}

Hepatic cysts are a common finding but their complications are rare. An infected hepatic cyst is an emergency and has to be treated in short terms. Modern imaging techniques help us to diagnose rapidly these lesions in the liver and, therefore, treat them.

Usually, the treatment consists of systemic antibiotics associated with abscess drainage.

This complication is still widely unknown but deserves more attention, due to the severe complications that can occur. It is necessary to rapidly recognize this condition to initiate an adequate treatment. Further studies would give us more epidemiological information.

\section{References}

1. ISHII K., YOSHIDA H., TANIAI N., MONETA S., KAWANO Y., TAJIRI T. Infected Hepatic Cyst Treated with Percutaneous Transhepatic Drainage. J. Nippon Med. Sch., 2009, 76 (3) : 160-164. doi : 10.1272/jnms.76.160.

2. MARUYAMA Y., OKUDA K., OGATA T., YASUNAGA M., ISHIKAWA H., HIRAKAWA Y., et al. Perioperative Challenges and Surgical Treatment of 
Large Simple, and Infectious Liver Cyst - A 12-Year Experience. PLoS ONE., 2013,8(10) : e76537. doi : 10.1371/journal.pone.0076537.

3. TELENTI A., TORRES V., GROSS JB., VAN SCOY RE., BROWN ML., HATTERY RR. Hepatic cyst infection in autosomal dominant polycystic kidney disease. Mayo Clin. Proc., 1990, 65(7) : 933-942. doi : 10.1016/ S0025-6196(12)65154-4.

4. AKOH JACOB A. Current management of autosomal dominant polycystic kidney disease. World Journal of Nephrology, 2015, 6 : 4 (4) : 468-479. doi : 10.5527/wjn.v4.i4.468.

5. FAROOQ Z., HESHMATZADEH BEHZADI A., BLUMENFELD J.D, ZHAO Y., PRINCE M.R. Complex Liver Cysts in Autosomal Dominant Polycystic Kidney Disease. Clin. Imaging, 2017, 46 : 98-101. doi : 10.1016/j. clinimag.2017.07.014.

6. MORII K., YAMAMOTO T., NAKAMURA S., OKUSHIN H. Infectious Hepa-tic Cyst : An Underestimated Complication. Intern. Med., 2018, 57 : 2123-2129. doi : 10.2169/internalmedicine.0511-17.

7. LANTINGA M.A., DRENTH J.P.H., GEVERS T.J.G. Diagnostic criteria in renal and hepatic cyst infection. Nephrol. Dial. Transplant., 2015, 30 : 744-751. doi: $10.1093 / \mathrm{ndt} / \mathrm{gfu} 227$

8. HENEGHAN H., HEALY A., MARTIN S., RYAN R., NOLAN N., TRAYNOR O., et al. Modern management of pyogenic hepatic abscess : a case series and review of the literature. BMC Research Notes, 2011, $4: 80$. doi: 10.1186/1756-0500-4-80.

9. LARDIÈRE-DEGUELTE S., RAGOT E., AMROUN K., PIARDI T., DOKMAK S., BRUNO O., et al. Hepatic abscess : Diagnosis and management. J. Visc. Surg., 2015, 152 (4) : 213-243. doi : 10.1016/j. jviscsurg.2015.01.013.
10. WIJNANDS TF., LANTINGA MA., DRENTH JP. Hepatic cyst infection following aspiration sclerotherapy : a case series. J. Gastrointestin. Liver Dis., 2014, 23 (4) : 441-444. doi : 10.15403/jgld.2014.1121.234.hcy.

11. MORTELÉ KJ., ROS PR. Cystic focal liver lesions in the adult : differential CT and MR imaging features. RadioGraphics, 2001, 21 (4) : 895-910. doi : 10.1148/radiographics.21.4.g01j116895.

12. YOSHIDA H., TAJIRI T., MAMADA Y., TANIAI N., KAWANO Y., MIZUGUCHI Y., et al. Infected Solitary Hepatic Cyst. J. Nippon Med. Sch., 2003, 70 (6) : 515-518. doi : 10.1272/jnms.70.515.

13. PRECETTI S., GANDON Y., VILGRAIN V. Imagerie des lésions kystiques du foie. J. Radiol., 2007, 88 (7-8 Pt 2) : 1061-1072. doi : 10.1016/s02210363(07)89919-7.

14. HOPE WW., VROCHIDES DV., NEWCOMB WL., MAYO-SMITH WW., IANNITTI DA. Optimal treatment of hepatic abscess. Am. Surg., 2008, 74 (2) 178-182.

15. RAJAK CL., GUPTA S., JAIN S., CHAWLA Y., GULATI M., SURI S. Percutaneous treatment of liver abscesses : needle aspiration versus catheter drainage. AJR Am. J. Roentgenol., 1998, 170 (4) : 1035-1039. doi : 10.2214/ ajr.170.4.9530055

16. MALIK AA., BARI SU., ROUF KA., WANI KA. Pyogenic liver abscess : Changing patterns in approach. World J. Gastrointest. Surg., 2010, 2(12) : 395-401. doi : 10.4240/wjgs.v2.i12.395.

17. CHICHE L., DARGËE S., LE PENNEC V., DUFAY C., ALKOFER B. Pyogenic-liver abscess : Diagnosis and management. Gastroenterol. Clin. Biol., 2008, 32 (12) : 1077-1091. doi : 10.1016/j.gcb.2008.09.019.

18. CHUNG SD., CHEN KH., CHANG HC. Pyelo-hepatic fistula. Urology, 2008, 72 (3) : 524. doi : 10.1016/j.urology.2008.02.075. 